\title{
L'homme des llanos
}

\author{
par R. FIASSON
}

L'homme des llanos, le "llanero » qui, comme le « gaucho », passe la plus grande partie de sa vie à cheval, est isolé dans sa pampa autant, sinon plus, que le marin sur l'océan. Son maître et ses compagnons de travail dans la ferme d'élevage que l'on appelle l' " hato » constituent souvent, et pour sa vie entière, ses uniques contacts avec l'humanité. Il ne pénètre dans la ville ou la bourgade qu'à de rares occasions, lors de la conduite des troupeaux de "novillos » (1) vers les montagnes de Colombie ou vers les abattoirs de Galabozo et de Maracay.

Ses plaisirs sont rares et simples. Un « asado » (rôti) de la viande bien grasse d'une génisse, de nombreuses tasses de café noir, très fort; parfois, mais peu souvent, un café «con leche »'et, le dimanche, s'it peut se l'offrir, une bouteille de mauvais rhum. Telles sont les joies du llanero.

Ses satisfactions, plutôt barbares, il les recherche dans l'exécution d'un travail dangereux. Domestiquer un cheval sauvage, réduire un taureau particulièrement agressif, chasser le jaguar à la lance, piéger le caïman, en somme exagerer le risque que la vie du llano lui apporte.

Telles sont les marques de l'homme de la pampa : brave, insouciant, d'une endurance à toute épreuve qui lui permet, le ventre creux à la fin d'une journée harassante de " rodeo », de chanter et de rire sous la pluie, au milieu de ses compagnons de peine.

Ses relations féminines se bornent à quelques misérables aventures avec la vieille cuisinière ou quelque ser'vante courbée sous les travaux de l'hato. Parfois, avec l'accord du maître (de l' « amo »), il invite à partager sa vie une jeune femme de ses brèves rencontres de la ville.

Demain, elle appartiendra à un autre et, si elle met au monde un enfant, celui-ci ne sera à personne, sinon anonymement à l'hato. Le « pourcentage

(1) Le "novillo» qui devrait être un bouvillon, représente ici tout animal cascré que l'on destine à la vente pour la boucherie. Il a souvent 7 ans ou davantage. des enfants légitimes dans les llanos doit être à peu près nul » a écrit Crist en 1935.

Mais un enfant, dans ces régions, est toujours un bienvenu. Il apporte une aide plutôt qu'une charge. Combien de couples que nous avons connus, couples temporaires bien entendu, et qui avaient autour d'eux une suite d'enfants qui n'appartenaient ni à l'homme ni à sa compagne.

Ces enfants ne peuvent pas s'instruire. Cela n'a aucune importance sociale car, dans ces pampas d'élevage, les enfants valent par eux-mêmes, par leur aptitude au travail de la ferme.

$\mathrm{L}$ analphabétisme doit atteindre au moins $90 \%$ de la population vénézuélienne.

Le « peon 》 (le serviteur) et l'amo (le maître) entretenaient, il n'y a pas si longtemps; des relations affectueuses. Il se créait dans la ferme des liens de famille plutôt que ceux qui unissent "Senor » et serviteurs, mais ces coutumes tendent à disparaître.

D'abord parce que dans la plupart des llanos vénézuéliens, le pétrole a fait éclater des coutumes qui réduisaient, malgré une apparence patriarcale, l'un à l'esclavage de l'autre. Cet esclavage économique qui se retrouve dans la plupart des pays d'Amérique latine, à évolution sociale peu avancée, est l'une des formes modernes de domination les plus difficilement supportables.

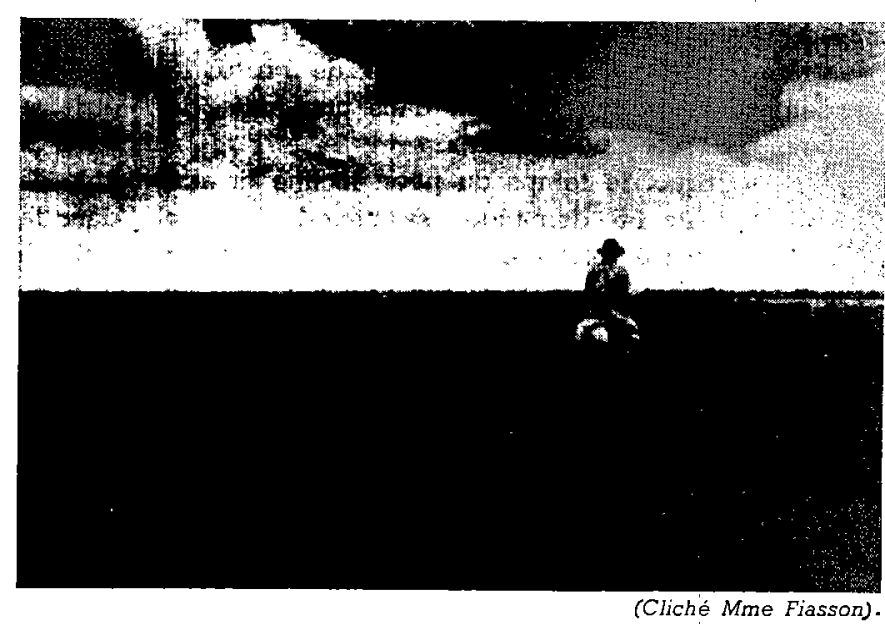

Llanero surveillant un troupeau. 


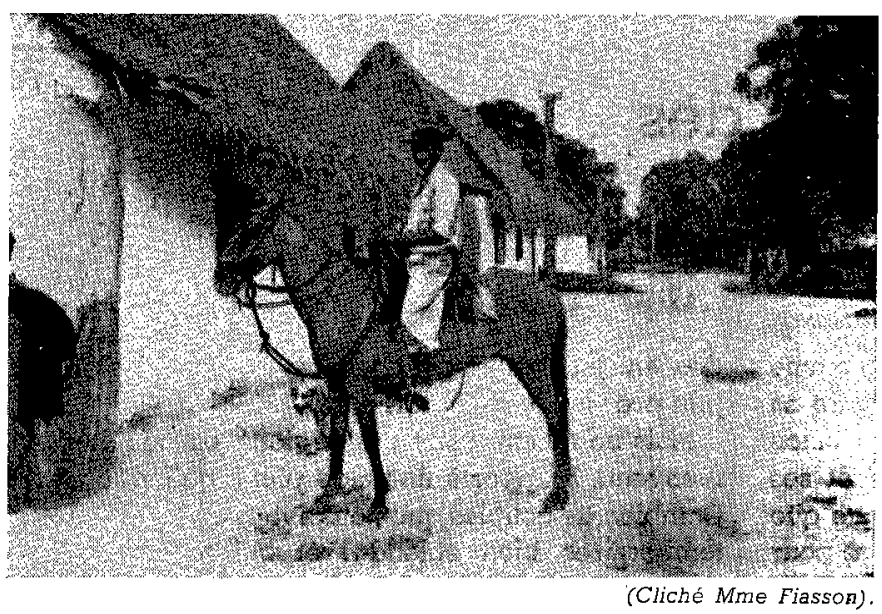

Llanero à cheval.

travail limitées, bref une vie moins sauvage, moins primitive, moins pénible.

Aussi la désertion a été rapide, profonde, inéluctable.

Et cet abandon a provoqué, en général, de profondes modifications de l'élevage dans les llanos.

Un nombre important de propriétaires qui, souvent d'ailleurs, se contentaient de recevoir l'afflux d'argent frais à chaque vente de novillos, c'est-à-dire au début et à la fin de la saison des pluies, et qui vivaient confortablement à Caracas, ou à Paris, ont préféré vendre leurs fermes et se créer d'autres sources de bénéfices plutôt que d'améliorer leurs méthodes de travail et réduire la main-d'œuvre.

Le peon de l'hato rcçoit le plus souvent un salaire dérisoire, inférieur au total de ses dépenses qui, ajoutons-le, se font uniquement dans la ferme où il travaille. Le propriétaire (ou le gérant), étant donné les distances qui le séparent de la ville et de ses magasins, a créé un centre d'approvisionnement : alcool, tabac, vêtements, savon, etc., à des prix beaucoup plus élevés que leur prix d'achat. Le serviteur ne pourrait acheter ailleurs ce qui lui manque.

Si l'on veut bien considérer, d'autre part, que le peon doit fournir sa selle, ses rênes, sa couverture de pluie (" cobija »), son hamac, en somme'ses instruments de travail, et qu'il aura aussi à assurer les frais du blanchissage de son linge, on comprendra qu'à la fin de l'année il est endetté à l'égard du maître, d'une sommo ćgale à deux ou trois fois la valeur de son salaire annuel.

Et lorsque ce serviteur, courbé par l'âge et la maladie, n'est plus d'aucune utilité, parfors le maître ose s'en séparer, le chasser de l'hato, le condamnant à mourir de faim dans une hutte de chaume, à l'orée d'un « mata ».

$C$ 'est pourquoi le temps du peon incliné devant le maître pour lui demander sa bénédiction est bien fini. Malgré la lenteur des communications et les formidables distances, la vie a évolué dans les pampas solitaires.

La difficulté qu'ont éprouvée les Compagnies pétrolières à trouver de la main-d'œuvre dans ces régions desertiques les a obligées à offrir des salaires trois à quatre fois plus élevés que ceux que les peons recevaient dans les fermes.

A ces offres tentantes (qui augmentent d'ailleurs rapidement), s'ajoutaient tous les avantages sociaux : contacts humains dans les cabarets, cinéma, relations féminines, nourriture plus variée, heures de
Ce sont alors des commerçants des petites bourgades voisinés qui ont acheté ces propriétés. Mais ces derniers n'avaient aucune préparation pour conduire de tels élevages et les relations de maîtres à serviteurs, déjà passablement autocratiques, sont devenues de barbares tentatives d'exploitation. L'élevage n'y a rien gagné.

Seuls quelques propriétaires qui vivaient sur l'hato ont su conserver un personnel qui leur était attaché sentimentalement (régions les plus éloignées de l'Apure et du Bolivar) et tentent maintenant d'améliorer leurs méthodes, de les rendre plus rationnelles.

\section{***}

La selle du llanero, fabriquée par des artisans locaux, est une simplification de la selle arabe, le cavalier étant seulement moins encastré, pommeau et troussequin étant moins élevés. Les étriers sont étroits et ne supportent que le gros orteil. Quelques selles mexicaines sont cependant utilisées avec des étriers de bois et un manchon dè cuir protégeant toute la partie antérieure du pied ce qui est très utile dans les déserts mexicains à cactus, mais pas dans les llanos.

Sur le côté gauche du pommeau est accrochée la « soga » (le lasso), lanière de cuir taillée dans la peau d'un boeuf en partant du centre : elle est ainsi d'une seule pièce et atteint 30 à $40 \mathrm{~m}$ de longueur. Elle est ensuite tannée à l'écorce de "dividivi » et assouplie avec de la graisse.

La tenue du llanero est sommaire. Le pantalon de toile roule au-dessus du genou; le "liquiliqui " (veste de 'toile) boutonné jusqu'au cou et un immense chápeau de feutre qui le protège de la pluie et du soleil. Pas de chemise ni de chaussures. La cobija est de règle pendant la saison des pluies. 
C'est une lourde couverhire de laine, rectangulaire, fendue sur $25 \mathrm{~cm}$, selon une diagonale, pour donner passage à la tête. Elle tombe en plis élégants tout autour du cavalier. Elle est imperméable et sera utilisée la nuit, au-dessus du hamac, pour servir de tente.

En dehors de cette couverture roulée en travers sur le troussequin, un sac de cuir étroit et allongé, accroché derrière la selle contient le hamac tissé (le " chinchorro ") et la moustiquaire indispensable à cause de la pullulation des insectes piqueurs et des vampires.

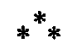

L'hato est une bâtisse massive, sans étage, à peine surélevée au-dessus de la plaine, construite en briques de boue séchée au soleil et armée de paille brisée ( " adobe ").

Il est formé d'une grande case rectangulaire dans laquelle sont délimitées 2 ou 3 pièces pour le propriétaire, sa famille et l'intendant. Les pieux qui soutiennent le toit sont taillés dans du bois dur que ni les termites ni l'humidité ne peuvent attaquer. L'armature légère du toit est recouverte de chaume ou de branches de palmiers quand on peut en trouver.

A proximite, une autre construction, plus petite, est utilisée comme cuisine et magasin. Enfin, d'un autre côté, une construction ne comportant souvent qu'un toit et ouverte sur les côtés (le " caney ») sert de refuge aux peons.

En général, le toit de la case principale est prolongé pour abriter une véranda, au moins sur l'une des façades principales.

Quelques propriétaires ont préféré les plaques de tôle au chaume, mais la demeure est beaucoup moins agréable et moins hygiénique.

Le lit est inconnu. Tout le monde dort dans des hamacs ( chinchorros ») qui comportent la moustiquaire. Le mobilier est réduit au strict minimum. Une table et des chaises en peau de bouf. Chacun possède une caisse pour mettre le linge à l'abri des « cucarachas » (cafards).

Ces habitations pullulent en général de rats, de chauve-souris, de cafards et de réduvidés (Triatoma et Rhodnius) dans le chaume du toit. Les insectes de cette dernière famille transmettent la maladie de Chagas.

Les conditions d'habitation du llanero (amo ou peon) sont donc extrêmement modestes. Mais dans le cadre d'une économie aussi primitive, la masure est normale. Où elle devient choquante et injustifiable, dans le même lieu de la pampa, c'est à partir du moment où le travailleur apporte son concours à une activité moderne. C'est le cas dans les Compagnies pétrolières qui dressent leurs derricks sur les llanos du Guarico, d'Anzoategui; de Monagas et de Barinas. A côté des bungalows de bois, surélevés, peints de couleurs agréables; formant des cilés modernes entourées d'un fossé rempli de pétrole brut, les ouvriers vénézuéliens ont bâti leurs cabanes de carton et de tôle qui, 'agglomérées, constituent d'invraisemblables Bidonvilles d'autant plus surprenants qu'ils ne s'adossent même pas à une agglomération importante qui pourrait les expliquer et les justifier en partie.

\section{**}

L emplacement de l'hato est toujours choisi sur une "medano » ou un "banco » qui ne s'inonde pas en hivernage.

Souvent le propriétaire a cherché à se rapprocher d'une " mata » afin de disposer d'uni terrain fertile où seront cultivés les bananes et le, manioc indispensables à la vie de la ferme.

Un hato dans les llanos comprend trois éléments principaux : la maison d'habitation que nous venons de décrire, les « corrals» pour le travail des animaux, un terrain clôturé pour les cultures vivrières.

La construction des corrals est l'objet de soins attentifs parce que de leur solidité dépendront le succès et la sécurité des travaux. futurs de l'hato. Ils sont délimités sur un terrain élevé, sec et dur. On coupe les pieux à l'époque du dernier quartièr de la lune, à la fin de l'hivernage, pour obtenir du bois ayant le moins de sève possible et profiter de l'interruption saisonnière des travaux ainsi que des facilités de transport par eau, les «'caños » étant en général pleins et les savanes inondées. Ces pieux sont profondément enterrés et solidement reliés les uns aux autres. On place les corrals à l'ouest de l'hato afin que la poussière soulevée par les déplacements des animaux ne soit pas rabattue sur les habitations par le vent du nord-est.

Au centre des corrals est la « majada » qui constitue l'enceinte la plus importante; et en communication avec celle-ci se trouvent trois, quatre ou cinq corrals secondaires selon l'importance des travaux à effectuer.

La majada doit être la plus solidement construite et assez étendue puisqu'elle doit pouvoir recevoir de grandes masses de novillos.

Les pieux y sont enfoncés de 60 à $80 \mathrm{~cm}$ de profondeur, serrés les uns contre les autres, sauf en certains endroits où des intervalles sont aménagés pour servir de refuge aux peons attaqués par les animaux. Dans un angle de la majada, près d'un de ces refuges où se placent les « mirones » qui font le calcul des animaux opérés, est solidement planté le "botalon », axe et base: de toutes les manœuvres. C'est un tronc de bois dur, très gros, 
enfoncé aussi profondément que possible dans le sol, terminé à son extrémité libre par une incision profonde sur laquelle passe et s'appuie la soga avec laquelle on saisit l'animal.

Beaucoup de propriétaires des hatos placent un véritable orgueil à posséder la plus grandé et la mieux construite des majadas. Dans certaines d'entre elles un troupeau de 800 à 1.000 animaux peut être enfermé et lorsqu'on a vu de quelles extraordinaires réactions sont susceptibles les taureaux llaneros récemment capturés, jamais encore dominés, lougueux et susceptibles, on ne manque pas d'être impressionné à les voir ainsi réduits à merci dans un corral.

Un hato de quelque importance possède d'autre part, dispersées sur l'étendue des terres, des dépendances que l'on appelle «fundacion » et qui constituent des centres de travail pour les peons; des corrals y sont aménagés et un toit de chaume leur pernet de s'abriter. Une fundacion est le plus souvent surveillée par un couple, de telle sorte que la femme peut subvenir aux repas des peons.

\section{***}

En fondant un hato, la première préoccupation du llanero est le « conuco » qui fournira le «pan », c'est-à-dire le champ où seront cultivés le « topocho" (variété de banane) et la « yuca » (manioc). Topocho et yuca sont effectivement le pain du llanero. Le topocho se mange coupé en tranches et frit, la yuca entière, bouillie ou rôtie. Le topocho, spécial au Venezuela, avec ses fruits triangulaires, courts, fait la transition entre le « plátano » (banane cochon) non sucré et le " camburo » qui contient au contraire beaucoup de sucre et peu d'amidon (1). Malheureusement le topocho est très attaqué par l' «ereque », maladie due à un champignon qui provoque la pourriture du tronc. Aussi, les plantations de 《 plàtano », plus résistant, remplacent-elles peu à peu celles de topocho.

Parfois une petite parcelle de haricots ( $\ll$ fri-

(1) « Plàtano» : Musa paradisiaca subsp. normalis (variétés : « comun », « dominico », « guineo», « chocheco »). Se mange cuit.

Composition : eau $15 \%$, amidon $66 \%$, sucre $1 \%$, autres substances (cellulose, etc.) $18 \%$.

« Banano 》 ou « Cambur »: Musa paradisiaca subsp. 2 sapientium (variétés : " guineo » [gros Michel], «cuyaco», «manzano», «cambur morado», « cambur rosado », « cambur resplandor », « titiaro $»)$.

Composition : eau $72 \%$, amidon $0 \%$, sucre $22 \%$, autres substances (cellulose, etc.), $6 \%$. joles »), de «quinchonchos " (Pois d'Angole : Cajanus indicus), « ñames 》, ignames (Dioscorea cayennensis); patates douces : "ocumo » (Xanthosoma sagittifolium); « guate» : Maranta arundinacea; « chayota »: Sechium edule. La culture de la canne à sucre s'est peu à peu développée afin de foumir la «panela » et le «papelon » dont le llanero est friand pour sucrer son café ou même tout simplement pour le dissoudre dans l'eau sous forme de " guarapo ». Le tabac est planté soit autour de la maison, soit dans les bois épais. Les feuilles, une fois séchées, sont roulées et transformées en tabac à chiquer avec du sucre et de la cendre. Il est ainsi vendu au mètre. Le llanero fume peu mais apprécie le tabac à mâcher, sans doute à cause de la facilité que lui apporte cette façon d'en user à cheval, sous la pluie, ou luttant avec un taureau.

Enfin, un petit champ de maïs permet, quand les fourmis parasols ou le « gusano cogollero » (la larve du cceur de la plante) ou les oiseaux prédateurs (perroquets verts), en ont laissé suffisamment, de confectionner la « cachapa » (galette de maïs tendre), sucrée et savoureuse, que tous les llaneros attendent avec impatience pour apporter une modification à un régime d'une sévère monotonie.

A côté des cultures vivrières existe parfois un " potrero », enclos ou l'on enferme les chevaux, aménagé en prairie artificielle d'herbe de Para.

\section{$*^{*} *$}

Nos expériences, conduites à l'Institut du llano, aux Bancos San-Petro, sur l'emplacement d'un ancien lit du Guarico (État Guarico), donc sur sol relativement riche (1), nous ont cependant montré la possibilité de cultures variées dans les llanos.

(1) Composition du sol selon les prélèvements effectués sur des parties surélevées et des parties inondées :

Entre 0 et $30 \mathrm{~cm}$ de profondeur :

Sable.................... 36 à $46 \%$ Argile................... 14,4, à $24,4 \%$ Limon $\ldots \ldots \ldots \ldots \ldots \ldots \ldots \ldots, 39,6$ à $49,6 \%$

Entre 30 et $60 \mathrm{~cm}$ de profondeur :

Sable................... 19 à $42 \%$ Argile.................. 24,4 à $35,4 \%$ Limon $\ldots \ldots \ldots \ldots \ldots \ldots \ldots \ldots \ldots$.............. 30 à $45,6 \%$

Ces terrains contiennent suivant les lieux considérés : Azote total $0,9-1,1-3$ pour mille; chaux 7,5- $11-13-26$ pour mille; acide phosphorique assimilable $8,5-4,1-10$ pour mille ; potasse assimilable 0 .

Le pourcentage en matière organique varie de 1,14 à 2,15 pour mille.

Le pH se maintient entre 5,7 et 6,2 . 
Le coton (variété locale), semé fin août, récolté en décembre, donna, avec un apport de superphosphate et de sulphosphate, un rendement égal à celui des champs d'expérience de la vallée de Aragua.

Les arachides (variété Jumbo Virginie), semées le 15 septembre, récoltées le 10 janvier : $3.500 \mathrm{~m}^{2}$ ont produit $380 \mathrm{~kg}$. Aucune différence de développement ni de rendement entre les parcelles avec engrais et celles sans engrais.

Kudzis tropical (Pueraria phaseoloídes) : résultats remarquables. Nous y reviendrons.

Canne à sucre (variété P. O. J. 2878) : se développa remarquablement et démontra une résistance parfaite à la sécheresse (sept mois sans eau).

Riz Zénith et Fortuna : semés au milieu d'août. Trop tard pour obtenir une récolte. Les rendements dans l'État de Portuguesa où plus de 10.000 ha ont été défrichés pour cette culture s'échelonnent entre 1.000 et $1.500 \mathrm{~kg}$ à l'ha selon le régime des pluies. De l'engrais y est apporté.'

Maïs (variétés vénézuéliennes) : bon développe ment et bonne récolte malgré l'abondance des perroquets verts. Mais c'est surtout en deuxième sole, sur des parcelles plantées de soja, de haricots et de sésame, que les résultats furent véritablement extraordinaires.

Soja (variété O-Too-Tan) : semé au début juillet, récolté début novembre. Résultats excellents. Une plantation de haricots fut faite sur le soja et eut le temps de parvenir à maturité avant l'établissement de la saison sèche.

Sésame (variété vénézuélienne) : semé $1^{\text {er }}$ juillet, récolté fin novembre. Résultats normaux.

Mil fourrager : semé le 15 octobre. Bon développement. Coupé comme fourrage vert. Arrosé en saison sèche.

Nous effectuâmes aussi des plantations de manioc, patate douce, igname, arrow-root, chayota, pois d'Angole, bananes dont les. résultats excellents étaient déjà connus des llaneros.

Les fèves "caraotas 》 y réussissent aussi, sauf les noires (Phaseolus vulgaris). Les haricots donnent partout des récoltes abondantes (Vigna).

Des essais de pommes de terre eurent un échec complet, mais, dans le jardin potager de l'Institut, tomates, salades variées, radis, choux-fleurs, chouxraves, haricots, carottes et surtout aubergines, donnèrent de très bons résultats.

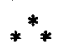

Un propriétaire aura d'autant plus de facilités à se procurer de la main-d'œuvre que son conuco sera plus prospere et ceci se comprend dans ces régions aussi éloignées et aussi isolées, même de nos jours, où l'avion évite un grand nombre de terrains des llanos durant tout l'hivernage.

Les récoltes un peu abondantes, faites par le llanero, ne sont done que de manioc et de bananes. Aussi, dans les llanos comme dans d'autres lieux dépourvus d'agriculture, l'alimentation est presque exclusivement carnée; Mongols, gauchos des solitudes argentines, Azzas du désert, Ténéré, etc.

Les hatos où l'on ne mange que de la viande ne sont pas si rares, viande fraiche, viande salée et séchée ( "tasajo ") que l'on réduit en poudre au moment de la préparer.

Ce mode obligé de nourriture ne doit pas être étranger à la résistance dont font preuve ces populations.

\section{$* *$}

Un certain souci d'orner les alentours de l'habitation se manifeste par des plantations de fleurs à la charge de la maitresse de maison. Il n'y a sans doute pas, en Amérique, un peuple aussi sensible aux beautés du règne végétal, aussi amoureux des fleurs que le peuple vénézuélien. Si sa demeure est sans confort, si le principal meuble de son logis est le hamac conjugal double, orné de franges et de pompons, la cour de la ferme présente, en général, un agréable parterre de fleurs multicolores et d'arbustes étranges. Bougainvillers appelés « trinitarias ", cheveux de Vénus, lianes corail, crotons, rosiers, amapolas et toutes sortes d'orchidées dont la plus répandue est la "flor de Mayo " (que le llanero rapporte des galeries forestières), plantes 
à parfums, s'y marient agréablement, bien que sans ordre.

C'est aussi à la femme qu'incombent les soins apportés aux arbres fruitiers, en particulier l'arrosage de chaque soir en saison sèche.

Grape-fruit, orangers, citronniers, anones diverses; « Chirimoya »': 'Anona cherimolia, « guanabana »: A. muricatus, « rinon»: $A$. cinerea, « anon »: A. squamma, la « parcha » ou barbadine : Passiflora quadrangularis, un ou deux spécimens de " onoto »: Bixa orellana, dont. la cuisinière ne saurait se passer pour colorer ses sauces et ses bouillons avec leș graines conservées dans de l'huile (1), " nispero »: Achras sapota, « tapara »: Crescentia cujete, dont l'écorce du fruit est utilisée pour la fabrication des calebasses, papayers, avocatiers, parfois deux ou trois cocotiers (arrosés au pelit lait ou à l'eau salée), anacardiers, manguiers enfin tels sont les arbres dont les feuilles bruissent sous la brise autour de l'hato.

Et la lutte ne cesse pas un instant pour les protéger de tous les ennemis qui les entotrent. Fourmis parasols (2) dont on tente d'arrêter l'ascension par une couronne de cheveux de femme brune (!), iguanes que l'on piege et que l'on mange (parfois on se contente de prélever leurs œufs par une incision ventrale et on leur redonne la. liberté), lézards, pucerons, chenilles, lièvres, tout un monde se ligue pour détruire une végétation bien trop rare pour qu'elle ne soit pas tentante.

Aussi le jardin pótager placé près de la cuisine, grand comme un drap de lit, est-il soigneusement installe sur pilotis. Sur une claie de branchages, entourées de murettes de boué séchée, les trois salades, les deux ciboulettes et la touffe de persil poussent sur $10 \mathrm{~cm}$ de terre, soigneusement pulvérisée et mélangée à du crottin dẹ cheval. Et c'est une chose touchante que de voir de combien d'amour et de soins empressés ces plantes fragiles sont entourées.

La culture esi difficile dans les llanos. Flle demande beaucoup d'efforts et elle n'est pas assurée d'une récolte. Ces fourmis parasols (" bachacas »), en particulier, causent de terribles ravages et leur élimination est un problème difficile à résoudre.

Aussi, bien que certaines régions, comme le

(1) C'est celle peinture ocre dont les indiens s'enduisent le corps.

(2) A Saint-Dominique, au commencement du $\mathrm{XVI}^{\circ}$ siècle, après avoir en vain brûlé les larves des fourmis, les moines conseillèrent aux habitants de choisir, par la voie du sort, un saint quii serait « abogado contra las hormigas ". L'honneur du choix tomba sur Saint-Saturnin.
Bas-Apure, sembleraient pouvoir constituer de magnifiques rizières, la plús grande partie de ce que le llanero mange en dehors de la viande, est importée. C'est d'ailleurs le drame actuel de: ce pays qui, ayant des revenus formidables que le pétrole lui apporte, ne trouve pas un équilibre suffisant pour réinvestir dans son sol ses énormes capitaux et les gaspille en achetant à l'étranger (à ceux-là mêmes qui lui versent ces dollars) tout ce qu'il peut consommer : viande des États-Unis et d'Argentine, sucre de Cuba, haricots du Chili, mangues de Trinidad, lait en poudre des Pays-Bas ou congelé d'Amérique du Nord, produits manufacturés du Nord-Amérique et de l'Europe. Le Venezuela n'exporte donc plus que son pétrole et le fer de sa Guyane.

Ce pays, dont la population n'atteint pas 4 millions d'habitants, tire $72 \%$ de son revenu du pétrolè qui, à son tour, représente $95 \%$ de ses exportations et ce pétrole esti pour la plus grande part, exporté et raffiné par des compagnies américaines.

Cette suprématie des U.S.A. au Venezuela augmente sans cesse depuis la découverte des minerais de fer (hématite) d'El Pao qui a motivé la création' d'un chemin de fer de $80 \mathrm{~km}$ qui la relie au port de San-Felix sur 1'Orénoque. La Bethleem Steel Corp. et la U.S. Steel Corp. les exploitent l'une et l'autre.

L'agriculture s'est malheureusement développée dans certains États des llanos d'une manière désordonnée et trop étendue, Dans l'État de Portuguesa, le Ministère de l'Agriculture aidé par la «Corporacion. Venezolona de Fomento " a constitué une massive politique du riz, défrichant des dizaines de milliers d'hectares de savanes pour les transformer en rizières. Le bétail, peu nombreux dans ces régions, a été parqué dans des « potreros comunales » (des enclos communaux) et ces vastes plaines au pied des Andes ont été livrées à la charrue sans aucune protection contre le vent qui emporte la terre en poussière et l'eau qui la pousse vers les affluents de l'Orénoque.

D'ailleurs, si les rizières ne couvrent pas encore l'Est de l'Apure; c'est tant mieux pour le bétail, car ces terres basses et fraîches forment de merveilleux pâturages de saison sèche pour tous les troupeaux qui descendent du Guarico brûlé.

Il eût suffi de peu de chose pour que cette politique du riz n'eût que des résultats heureux. Obliger les bénéficiaires des crédits à planter en deuxième année le tiers de leurs terrains défrichés en prairies artificielles et couper ces étendues par des lignes arbustives brise-vent dirigées du nord-ouest au sud-est. Mais qui y penserait dans ce pays des décisions incontrôlées? 
Il existe aussi des Indiens dans les llanos vénézuéliens, mais ceux-ci n'intéressent pas la communauté. Les rapports entre eux et les éleveurs sont généralement empreints de la pire des brutalités. L'Indien que l'on appelle dédaigneusement « bravo " (sauvage) a compris depuis longtemps qu'il ne devait attendre aucun soulagement à sa misère de la part de son compagnon des plaines, aussi misérable, aussi ignorant, mais qui, lui, s'enorgueillit du titre de « racional » (raisonnable).

Il n'existe d'ailleurs, dans ces distinctions, aucune idée de racisme. Le sang du llanero est beaucoup plus près de celui de son ancêtre, l'esclave africain, que de celui du conquistador espagnol. Les habitants des plaines, à part quelques rares exceptions de propriétaires, forment la grande famille de la race " brune " comme l'appellait un président de la République de Colombie. Et beaucoup d'entre eux ont des traces fort apparentes de sang indien, de ce sang indien pour lequel ils n'ont que haine et mépris.

A.ussi l'Indien s'est-il réfugié derrière les barrières virtuelles des cours d'eau et encore n'y peut-il vivre qu'en faisant régner la terreur dans son domaine.

Il attaquera le racional qui s'y aventure et celui-ci, à son tour, y effectuera de terribles expéditions punitives pour protéger ses gens et ses troupeaux. Voilà pourquoi d'immenses étendues d'herbages atteignant sans doute $20.000 \mathrm{~km}^{2}$ sont inexploitées, vides d'animaux, dans le sud de l'Apure, depuis l'Arauca jusqu'au Meta.

Aucun souci ne s'est d'ailleurs manifesté pour tenter de faire de ces populations des collaboratrices de l'économie vénézuélienne. Les Indiens Yaruro sont actifs et accueillants. Certains d'entre eux sont d'ailleurs employés comme peons dans les hatos. Quelques groupements installés sur la Capanaparo cultivent le manioc, la canne à sucre, les bananes. En novembre, ils sèment dans les bas-fonds et ils récoltent en mai. Ils effectuent alors de nouvelles cultures sur les hauteurs. Ils défrichent en général sur les rives des cours d'eau ce qui leur permet de visiter en pirogues leurs champs qui sont parfois très éloignés de leur résidence. Ainsi les Yaruro de Vuelta-Mala ont leurs plantations de cannes sur le Riecito, à trois jours de navigation.

Avec le manioc amer ils confectionnent les immenses galettes plates et dures, que l'on nomme "Casabe " el qui sont un pain très apprécié de tous les habitants des llanos.

On râpe le tubercule, puis on le presse fortement dans un appareil de joncs tressés. On laisse ensuite précipiter l'amidon et le résidu est desséché sous forme de galettes.

Les loisirs que leurs cultures et la pêche leur laissent sont employés à la fabrication des hamacs qu'ils tissent avec des fibres du palmier morichal (Mauritia flexuosa).

Les seules viandes de leur ration sont fournies par les tortues et les poissons, parfois le gibier. Mais ces Indiens s'intéresseraient à l'élevage s'ils avaient la possibilité de se procurer des animaux. Or, entre le Capanaparo et le Cinaruco existent d'immenses et excellents pâturages a'bondamment arrosés.

Des éleveurs ont tenté de fonder des fermes dans ces territoires inoccupés ( premiere conséquence est la diminution du gibier qui, pour beaucoup de tribus, constitue, avec le poisson et quelques produits de cueillette, leur seule nourriture. Or, les bœufs qui l'ont remplacé sont tout aussi sauvages; beaucoup d'entre eux ne portent pas de marques. La mentalite primitive de l'Indien ne peut faire de différence entre cette vache et le cerf ou le grand cabiais qui, avant l'arrivee du racional constituaient son gibier. Il l'abattra pour se nourrir, n'ayant même pas le sentiment de commettre un acte répréhensible. Quant au propriétaire il ne saurait admettre que des gens qui ne lui rendent aucun service prélèvent un tribut sur son troupeau.

Au sud de l'Arauca, entre certaines tribus indiennes et des propriétaires, se sont déclarées des luttes d'une telle violence qu'il est difficile de prévoir comment elles peuvent prendre fin.

\section{$*^{*} *$}

Les philosophes pourront se consoler en pensant que ces Indiens ont été aussi des envahisseurs. Ce sont sans doute des descendants des Caribes, venus des îles des Antilles un ou deux siècles avant les Espagnols, et qui avaient conquis le pays en suivant les chemins des eaux, de l'Orénoque et de ses affluents. Ils avaient détruit la civilisation Arawak dont on peut retrouver quelques vestiges dans les llanos.

Verneau avait déjà signalé d'antiques sépultures dans la région de l'Árauca. Nous avons, nousmême, mis à jour de superbes urnes funéraires sur les rives de ce cours d'eau. Elles ont $75 \mathrm{~cm}$ de hauteur, un diamètre sensiblement égal et contiennent des squelettes accroupis aux crânes déformés.

Sur les bords de l'Apure, un autre cimetière montre des urnes petites, rondes, qui renferment des crânes. Nous avons aussi répéré et signalé treize « medanos » (ou « mounds ») dans les pampas du Haut-Apure, restes d'anciens villages, d'où nous avons rapporté des morceaux de poterie, des statuettes en terre cuite et deux très curieuses lampes, taillées dans de la pierre de jais. 


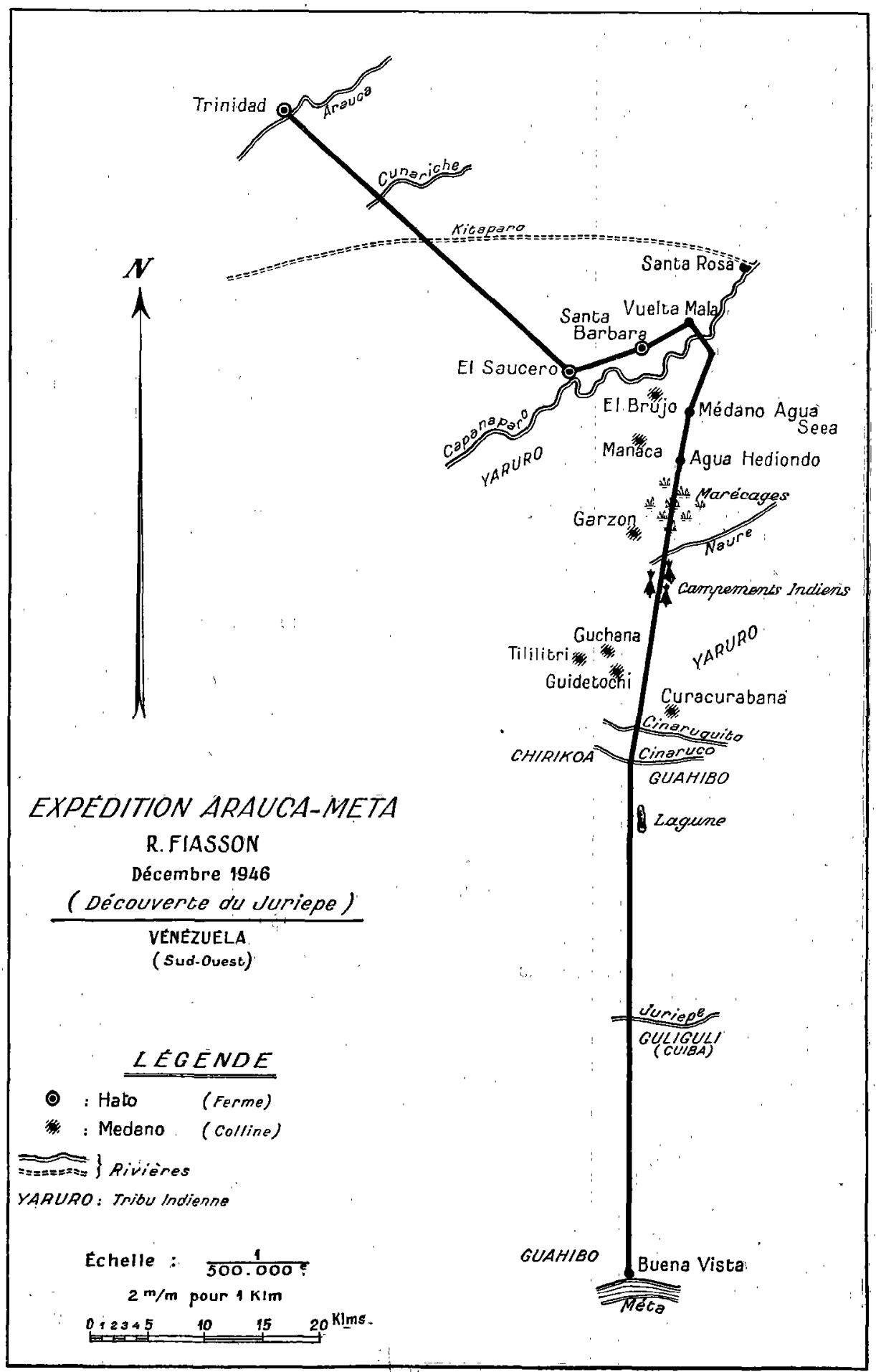


Parfois, deux de ces médanos sont réunis par une digue appelée "lomo de perro » (dos de chien). Dans d'autres régions ces digues forment des chaussées surélevées (des « calzadas 》) de 3 a $4 \mathrm{~m}$ au dessus du niveau de la plaine, larges d'une douzaine de mètres et se prolongeant sur plusieurs kilomètres.

Ces constructions, qui exigeaient une main-d'œuvre d'esclaves dans une organisation sociale élevée, dans un état fort, pouvaient être des voies sacrées. Dans tous les cas, elles sont si étranges et posent un si curieux problème qu'on a appelé les Arawak, les "Calzadas Builders ».

C'est cette civilisation que l'on suppose avoir été détruite par les Caribes. Ceux-ci d'ailleurs effectuèrent encore des incursions, en 1578 et 1580 . le long des rives du Guarico. Elles furent repoussées par les Espagnols.

Mais les tribus sauvages, encore existantes dans - les llanos, posent des problèmes qui, en l'absence de toute étude, ne seront pas résolus de sitôt.

Les cartes signalent toujours des indiens Otomacs que nous n'avons jamais vus. Dans le sud de l'Apure se rencontrent, en dehors des Yaruro très sociables, les Guahibo, les Chirikoa avec lesquels le voyageur peut prendre contact sans trop de risques.

Les Saliba, aux mœurs efféminées, sont aussi, dans certaines conditions, abordables, mais les Amorua qui effectuent des migrations saisonnières considérables qui les amènent du Rio-Negro au Meta, à la recherche, selon les saisons, de fruits et d'œufs de tortue, ne sont fréquentables que pendant le jour. Il vaut mieux éviter de dormir au milieu d'eux sur les rives du Meta. Enfin les Cuiba (ou Guliguli) font régner la terreur dans les parties les plus reculées de cette région. Les autres indiens en parlent comme de bêtes féroces. Nous n'en avons malheureusement pas rencontré bien qu'ayant suivi les rives du Juriepe, qui constituent leurs repaires, cours d'eau qu'aucune carte ne mentionnait avant notre exploration.

\section{$*^{*} *$}

Les villages à l'intérieur de ces vastes plaines sont rares. Encore les trouve-t-on surtout en bordure des cours d'eau, où les communications ne seront pas interrompues en saison des pluies.

Partout ailleurs ne sont plus que des étendues de savanes partagées entre les hatos. Ainsi, dans tout l'État de l'Apure, seul Mantecal est un centre créé en dehors du chemin des eaux, ce qui d'ailleurs ne facilite pas son accès en hivernage, et les $300 \mathrm{~km}$ qui le séparent de San-Fernando, la capitale de l'État, demandent souvent une dizaine de jours.

Les terrains communaux de ces bourgades sont en général extrêmement étendus $(50.000$ ha pour Mantecal), et constituent des pâturages pour tous ceux qui ne sont pas propriétaires de terrains et qui élèvent des troupeaux. Une fois l'an, les proprietaires, dont les troupeaux sont assez importants, font, à cheval et au lasso, la chasse aux animaux non marqués et se les approprient en y apposant leur fer.

Ces villages perdus dans la pampa sont en général protégés de l'incursion des animaux qui, pendant la nuit, ont tendance à rechercher des terrains secs où les insectes vulnérants sont moins nombreux.

Une haie de fils de fer ronce entoure donc la population et les sorties sont assurées par des " mata-burros » (que les Américains appellent " grid »). Ce sont des fossés qui coupent la route au niveau des barrières, sur lesquels des rails ont été disposés de telle façon que les véhicules et les gens franchissent le passage sans difficultés, alors que les animaux ne peuvent s'y aventurer.

Ce système est plus sûr que les portes que l'on oublie régulièrement de fermer.

Cette barrière est utilisée aussi lorsqu'une route coupe une haie sur la savane d'un hato.

Ainsi le silence des nuits llaneras n'est plus troublé que par le crissement des grillons ou le brame lointain de quelque bovin perdu dans l'immensité de la savane et qui réclame ses compagnons de troupeau. La brume impalpable qui, en saison seche, enveloppe la plaine après les tourbillons du vent du jour, fait place, en hivernage, au crépitement incessant de la pluie qui remplit les lagunes. Le concert de la nuit est alors renforcé par les cris d'une toute petite grenouille; cris rauques, immenses, qui, bien que lancés de tout près, font penser à des appels humains angoissés surgissant du fond de l'horizon. 